IPM/P-2002/007

hep-th/0204117

\title{
Gravity on noncommutative D-branes
}

\author{
F. Ardalan, ${ }^{a, b, 1}$ H. Arfaei,$^{a, b, 2}$ M. R. Garousi ${ }^{a, c, 3}$ and A. Ghodsi ${ }^{a, b, 4}$ \\ a Institute for Studies in Theoretical Physics and Mathematics (IPM), \\ P.O. Box 19395-5531, Tehran-Iran \\ b Department of Physics, Sharif University of Technology, \\ P.O. Box 11365-9161, Tehran-Iran \\ ${ }^{c}$ Department of Physics, Ferdowsi University, Mashhad-Iran
}

\begin{abstract}
The effective action for the low energy scattering of two gravitons with a D-brane in the presence of a constant antisymmetric $B$ field in bosonic string theory is calculated and the modification to the standard D-brane action to first order in $\alpha^{\prime}$ is obtained.
\end{abstract}

\footnotetext{
${ }^{1}$ Ardalan@theory.ipm.ac.ir

${ }^{2}$ Arfaei@theory.ipm.ac.ir

${ }^{3}$ Garousi@theory.ipm.ac.ir

${ }^{4}$ Ahmad@theory.ipm.ac.ir
} 


\section{Introduction}

The discovery in the early days of string theory that string amplitudes in low energy regimes maybe reproduced by Yang-Milles field theory for open strings and gravitational field theory for closed strings, was the beginning of a long, fruitful study into the relation of string theory and field theory in general and in low energy in particular $[1,2]$.

The lowest order in momentum expansion of the low energy string theory confirmed the interpretation of the massless string modes as the gauge bosons and gravitons of the respective theories, and the next order was a prediction for the corrections to the gauge theory and to the gravity $[3,4]$.

The realization of the significance of D-branes in string theory, correspondingly led to the study of the low energy effective dynamics of these objects in addition to the bulk dynamics $[5,6,7,8,9,10,14]$. The lowest order in the momentum expansion turned out to be the DBI action with higher order corrections involving nontrivial terms, both in the bosonic string and the superstring theory.

Discovery of noncommutativity in string theory in the presence of a nontrivial background [11] resulted in the dramatic resurgence of noncommutative gauge theories with their highly nontrivial novel properties emerging as deformation of the low energy action of open strings in the presence of a background. Higher order corrections to the noncommutative gauge theory have also been derived.

It is then natural to ask what kind of deformation the low energy effective action of closed strings suffers in the presence of constant back ground antisymmetric field. Noncommutative deformations of gravity have been proposed and studied recently [12]. However they have not been derived from string theory and are ad hoc in this sense. One of the motivations of the present study is to throw light on these deformations.

In this work we begin to address this problem by looking at the low energy amplitude of two closed strings with a D-brane in the presence of a constant background $B$ field. We find that as expected the bulk action is not modified, however, the effective action describing the dynamics of the D-brane is modified due to the presence of the constant B field on the D-brane. However the effective action is not obtainable, as a naive T-duality might indicate, from the action of vanishing $B$, by replacing the metric $g$ by $g+B$.

This might be understood on the basis of the fact that T-duality, at this order, mixes graviton scattering with antisymmetric field scattering and is highly nontrivial.

In section two we write down the amplitude of scattering of two gravitons with a D-brane in the presence of a B field and find its low energy behaviour. In section three we find the low energy effective action describing those amplitude to order $\alpha^{\prime}$. Section four is devoted to discussion, in particular on the issue of covariance of the effective action. 


\section{String scattering from D-branes}

In this section we compute the tree level bosonic string scattering amplitude of two massless closed string off a noncommutative D-brane. We take D-brane as a disk and conformaly mapped on the upper half plane, with mixed boundary conditions and use the following notation for indices, on and off the D-brane

$$
\mu, \nu=0, . ., 25 \quad, \quad a, b=0, . ., p \quad, \quad i, j=p+1, . ., 25 .
$$

For a D-brane localized at $x^{p+1}, \ldots, x^{25}$, the boundary conditions are

$$
\eta_{a b}(\partial-\bar{\partial}) X^{a}+\left.B_{a b}(\partial+\bar{\partial}) X^{a}\right|_{z=\bar{z}}=0
$$

The two points correlator of string coordinates $X^{\mu}(z, \bar{z})$ on the D-brane is

$$
\left\langle X_{i}^{\mu} X_{j}^{\nu}\right\rangle=\frac{-\alpha^{\prime}}{2}\left[\eta^{\mu \nu} \log \left(\left(z_{i}-z_{j}\right)\left(\bar{z}_{i}-\bar{z}_{j}\right)\right)+D^{\mu \nu} \log \left(z_{i}-\bar{z}_{j}\right)+D^{\nu \mu} \log \left(\bar{z}_{i}-z_{j}\right)\right]
$$

where

$$
D^{b a}=2\left(\frac{1}{\eta+B}\right)^{a b}-\eta^{a b} \quad, \quad D^{i j}=-\delta^{i j} \quad, \quad D_{\alpha}^{\mu} D^{\nu \alpha}=\eta^{\mu \nu}
$$

with $g$ the flat metric. Using these correlators we can compute the scattering amplitude of two closed strings off a D-brane,

$$
A=g_{c}^{2} e^{-\lambda} \int d^{2} z_{1} d^{2} z_{2}\left\langle V\left(z_{1}, \bar{z}_{1}\right) V\left(z_{2}, \bar{z}_{2}\right)\right\rangle
$$

with $g_{c}$ the closed string coupling constant and $\lambda$ the Euler number of the world sheet. The appropriate vertex operators for the closed strings are,

$$
V\left(z_{i}, \bar{z}_{i}\right)=\epsilon_{\mu \nu}: \partial X^{\mu}\left(z_{i}\right) \exp \left(i k_{i} \cdot X\left(z_{i}\right)\right):: \bar{\partial} \widetilde{X}^{\nu}\left(\bar{z}_{i}\right) \exp \left(i k_{i} \widetilde{X}\left(\bar{z}_{i}\right)\right):
$$

which the momenta $k_{i}$ and polarizations $\epsilon_{\mu \nu}$ of the massless closed strings satisfying

$$
\epsilon_{\mu \nu} k^{\mu}=\epsilon_{\mu \nu} k^{\nu}=0 \quad, \quad k^{2}=0 .
$$

To go further we use the change of variable as follow,

$$
\widetilde{X}^{\mu}\left(\bar{z}_{i}\right)=D_{\xi}^{\mu} X^{\xi}\left(\bar{z}_{i}\right)
$$

so that the closed string vertices change to

$$
V\left(z_{i}, \bar{z}_{i}\right)=\epsilon_{\mu \lambda} D_{\nu}^{\lambda}: \partial X^{\mu}\left(z_{i}\right) \exp \left(i k_{i} \cdot X\left(z_{i}\right)\right):: \bar{\partial} X^{\nu}\left(\bar{z}_{i}\right) \exp \left(i k_{i} \cdot D \cdot X\left(\bar{z}_{i}\right)\right):
$$

Now inserting these vertices into (5) and going through the calculation of the correlation functions we find

$$
A=g_{c}^{2} e^{-\lambda} \epsilon_{1 \mu \lambda} D_{\nu}^{\lambda} \epsilon_{2 \alpha \rho} D_{\beta}^{\rho} \int d^{2} z_{1} d^{2} z_{2} \mathcal{A}^{\mu \nu \alpha \beta}
$$


where

$$
\begin{aligned}
\mathcal{A}^{\mu \nu \alpha \beta} & =i C_{D_{2}}^{X}(2 \pi)^{p+1} \delta^{p+1}\left(k_{1}+k_{1} \cdot D+k_{2}+k_{2} \cdot D\right) \exp \left\{\frac { \alpha ^ { \prime } } { 2 } \left[k_{1} \cdot D \cdot k_{1} \log \left(z_{1}-\bar{z}_{1}\right)\right.\right. \\
& +k_{2} \cdot D \cdot k_{2} \log \left(z_{2}-\bar{z}_{2}\right)+k_{1} \cdot k_{2} \log \left(z_{1}-z_{2}\right)+k_{1} \cdot k_{2} \log \left(\bar{z}_{1}-\bar{z}_{2}\right) \\
& \left.\left.+k_{2} \cdot D \cdot k_{1} \log \left(z_{1}-\bar{z}_{2}\right)+k_{1} \cdot D \cdot k_{2} \log \left(\bar{z}_{1}-z_{2}\right)\right]\right\} \\
& \times\left\{q_{1 \overline{1}}^{\mu \nu} q_{2 \overline{2}}^{\alpha \beta}+q_{12}^{\mu \alpha} q_{\overline{1} \overline{2}}^{\nu \beta}+q_{1 \overline{2}}^{\mu \beta} q_{\overline{1} 2}^{\nu \alpha}+q_{1 \overline{1}}^{\mu \nu} f_{2}^{\alpha} f_{\overline{2}}^{\beta}+q_{12}^{\mu \alpha} f_{\overline{1}}^{\nu} f_{\overline{2}}^{\beta}+q_{1 \overline{2}}^{\mu \beta} f_{\overline{1}}^{\nu} f_{2}^{\alpha}\right. \\
& \left.+q_{\overline{1} 2}^{\nu \alpha} f_{1}^{\mu} f_{\overline{2}}^{\beta}+q_{\overline{1}}^{\nu \beta} f_{1}^{\mu} f_{2}^{\alpha}+q_{2 \overline{2}}^{\alpha \beta} f_{1}^{\mu} f_{\overline{1}}^{\nu}+f_{1}^{\mu} f_{\overline{1}}^{\nu} f_{2}^{\alpha} f_{\overline{2}}^{\beta}\right\}
\end{aligned}
$$

in which $C_{D_{2}}^{X}$ is the functional determinant for the string fields and the delta function ensures conservation of momentum parallel to the D-brane. Here $f$ 's are terms that come from correlation function between derivative and exponential terms while the $q$ 's are between the derivative terms, and are

$$
q_{i j}^{\mu \nu}=-\frac{\alpha^{\prime}}{2} \frac{\eta^{\mu \nu}}{\left(z_{i}-z_{j}\right)^{2}} \quad, \quad f_{i}^{\mu}=-i \frac{\alpha^{\prime}}{2} \sum_{j \neq i}\left(\frac{k_{j}^{\mu}}{z_{i}-z_{j}}\right),
$$

with the indices as

$$
i, j=1, \overline{1}, 2, \overline{2} \quad, \quad z_{\overline{1}}=\bar{z}_{1} \quad, \quad z_{\overline{2}}=\bar{z}_{2} .
$$

It is straight forward to see that the amplitude is $\mathrm{SL}(2, \mathrm{R})$ invariant. To fix this invariance we let $z_{1}=i y$ and $z_{2}=i$

$$
d^{2} z_{1} d^{2} z_{2} \rightarrow 2\left(1-y^{2}\right) d y
$$

The $f$ and $q$ terms then become

$$
\begin{aligned}
& f_{1}^{\mu}=\frac{1}{2 i y}\left(\frac{y+1}{y-1} k_{2}^{\mu}+\frac{y-1}{y+1} k_{2} \cdot D^{\mu}\right) \quad, \quad f_{\overline{1}}^{\nu}=\frac{-1}{2 i y}\left(\frac{y-1}{y+1} k_{2}^{\nu}+\frac{y+1}{y-1} k_{2} \cdot D^{\nu}\right) \\
& f_{2}^{\alpha}=\frac{-1}{2 i}\left(\frac{y+1}{y-1} k_{1}^{\alpha}+\frac{y-1}{y+1} k_{1} \cdot D^{\alpha}\right) \quad, \quad f_{\overline{2}}^{\beta}=\frac{1}{2 i}\left(\frac{y-1}{y+1} k_{1}^{\beta}+\frac{y+1}{y-1} k_{1} \cdot D^{\beta}\right) \\
& q_{1 \overline{1}}^{\mu \nu}=\frac{-\eta^{\mu \nu}}{4 y^{2}}, \quad q_{12}^{\mu \alpha}=\frac{-\eta^{\mu \alpha}}{(y-1)^{2}} \quad, \quad q_{1 \overline{2}}^{\mu \beta}=\frac{-\eta^{\mu \beta}}{(y+1)^{2}} \\
& q_{12}^{\nu \alpha}=\frac{-\eta^{\nu \alpha}}{(y+1)^{2}} \quad, \quad q_{\overline{1} \frac{\nu}{2}}^{\nu \alpha}=\frac{-\eta^{\nu \beta}}{(y-1)^{2}} \quad, \quad q_{2 \overline{2}}^{\alpha \beta}=\frac{-\eta^{\alpha \beta}}{4} .
\end{aligned}
$$

We have dropped terms which will be canceled when inserted in (10) using the physical conditions for closed strings. Inserting (15) into (11) we get

$$
A=2 i \mathcal{C} 2^{\frac{\alpha^{\prime}}{2}\left(k_{1} \cdot D_{S} \cdot k_{1}+k_{2} \cdot D_{S} \cdot k_{2}\right)} \int_{0}^{1} d y\left(1-y^{2}\right) y^{\frac{\alpha^{\prime}}{2} k_{1} \cdot D_{S} \cdot k_{1}}(y-1)^{\alpha^{\prime} k_{1} \cdot k_{2}}(y+1)^{\alpha^{\prime} k_{1} \cdot D_{S} \cdot k_{2}}
$$




$$
\begin{aligned}
& \left\{\left(\frac{\alpha^{\prime}}{2}\right)^{2}\left[\frac{a_{1}}{16 y^{2}}+\frac{a_{2}}{(y-1)^{4}}+\frac{a_{3}}{(y+1)^{4}}\right]+\left(\frac{\alpha^{\prime}}{2}\right)^{3}\left[-\frac{a_{4}}{16 y^{2}}+\frac{a_{5}}{4 y(y+1)^{2}}\right.\right. \\
& \left.-\frac{a_{6}}{4 y(y-1)^{2}}-\frac{a_{7}(y+1)^{2}}{16 y^{2}(y-1)^{2}}-\frac{a_{8}(y-1)^{2}}{16 y^{2}(y+1)^{2}}+\frac{a_{9}(y-1)^{2}}{4 y(y+1)^{4}}-\frac{a_{10}(y+1)^{2}}{4 y(y-1)^{4}}\right] \\
& \left.+\left(\frac{\alpha^{\prime}}{2}\right)^{4}\left[\frac{a_{11}}{16 y^{2}}+\frac{a_{12}(y+1)^{2}}{16 y^{2}(y-1)^{2}}+\frac{a_{13}(y-1)^{2}}{16 y^{2}(y+1)^{2}}+\frac{a_{14}(y+1)^{4}}{16 y^{2}(y-1)^{4}}+\frac{a_{15}(y-1)^{4}}{16 y^{2}(y+1)^{4}}\right]\right\},
\end{aligned}
$$

where

$$
\mathcal{C}=C_{D_{2}}^{X} g_{c}^{2} e^{-\lambda}(2 \pi)^{p+1} \delta^{p+1}\left(k_{1}+k_{1} \cdot D+k_{2}+k_{2} \cdot D\right) .
$$

$D_{A}$ and $D_{S}$ are the antisymmetric and symmetric parts of $D$ matrix. Notice that because of momentum conservation parallel to the D-brane we have $k_{1} \cdot D_{A} \cdot k_{2}=0$. The constants $a_{n}$ 's depend on momentum and polarization of external states and are given in the appendix A. We choose the Mandelstam variables,

$$
\begin{aligned}
& s=-\left(\frac{1}{2}(\eta+D) \cdot k_{1}\right)^{2}=-\frac{1}{2} k_{1} \cdot D_{S} \cdot k_{1}=-\frac{1}{2} k_{2} \cdot D_{S} \cdot k_{2}, \\
& t=-\left(k_{1}+k_{2}\right)^{2}=-2 k_{1} \cdot k_{2} .
\end{aligned}
$$

Finally making the change of variable $y=\frac{1-\sqrt{x}}{1+\sqrt{x}}$ we find

$$
\begin{aligned}
A & =2 i \mathcal{C}\left(\frac{\alpha^{\prime}}{2}\right)^{2}\left\{a_{1} B\left(-\frac{\alpha^{\prime} t}{4}+1,-\alpha^{\prime} s-1\right)+a_{2} B\left(-\frac{\alpha^{\prime} t}{4}-1,-\alpha^{\prime} s+1\right)\right. \\
& +a_{3} B\left(-\frac{\alpha^{\prime} t}{4}+1,-\alpha^{\prime} s+1\right)-\left(\frac{\alpha^{\prime}}{2}\right)\left[a_{4} B\left(-\frac{\alpha^{\prime} t}{4}+1,-\alpha^{\prime} s-1\right)\right. \\
& -a_{5} B\left(-\frac{\alpha^{\prime} t}{4}+1,-\alpha^{\prime} s\right)+a_{6} B\left(-\frac{\alpha^{\prime} t}{4},-\alpha^{\prime} s\right)+a_{7} B\left(-\frac{\alpha^{\prime} t}{4},-\alpha^{\prime} s-1\right) \\
& +a_{8} B\left(-\frac{\alpha^{\prime} t}{4}+2,-\alpha^{\prime} s-1\right)-a_{9} B\left(-\frac{\alpha^{\prime} t}{4}+2,-\alpha^{\prime} s\right) \\
& \left.+a_{10} B\left(-\frac{\alpha^{\prime} t}{4}-1,-\alpha^{\prime} s\right)\right]+\left(\frac{\alpha^{\prime}}{2}\right)^{2}\left[a_{11} B\left(-\frac{\alpha^{\prime} t}{4}+1,-\alpha^{\prime} s-1\right)\right. \\
& +a_{12} B\left(-\frac{\alpha^{\prime} t}{4},-\alpha^{\prime} s-1\right)+a_{13} B\left(-\frac{\alpha^{\prime} t}{4}+2,-\alpha^{\prime} s-1\right) \\
& \left.\left.+a_{14} B\left(-\frac{\alpha^{\prime} t}{4}-1,-\alpha^{\prime} s-1\right)+a_{15} B\left(-\frac{\alpha^{\prime} t}{4}+3,-\alpha^{\prime} s-1\right)\right]\right\},
\end{aligned}
$$

where $B$ 's are beta functions. Note that as a test of our calculations replacing every polarization of closed strings by momentum $\epsilon_{\mu \nu} \rightarrow k_{\mu} k_{\nu}$ gives zero value for the amplitude. We recover the results of scattering amplitude for commutative D-branes found in [14] by turning the $B$ field off (see Appendix B). As we will be interested in the low energy effective theory of the string, we must analyze the pole structure 
of the amplitude (19). There are two types of singularities in this amplitude that come from the Beta functions. In fact the amplitude (19) is singular at

$$
-\frac{\alpha^{\prime}}{4} t-1=-n \quad, \quad-\alpha^{\prime} s-1=-n^{\prime} \quad, \quad n, n^{\prime}=0,1, \ldots
$$

which in terms of $t$ and $s$ of (18) are

$$
\begin{aligned}
& t=m_{\text {closed }}^{2}=-\frac{4}{\alpha^{\prime}}(-n+1) \\
& s=m_{\text {open }}^{2}=-\frac{1}{\alpha^{\prime}}\left(-n^{\prime}+1\right),
\end{aligned}
$$

exhibiting the mass spectrum of the intermediate closed string propagators in the t-channel and the mass spectrum of the open strings in the s-channel. Note that not all the Beta functions in (19) have pole for $n=0,1,2,3$ and $n^{\prime}=0,1$. Now we may study the low energy limit of the amplitude i.e. $s, t \rightarrow 0$. There are then three types of terms to consider

t - channel :

$$
A_{t}=\frac{i \mathcal{C} \alpha^{\prime 2}}{t}\left\{-2 a_{2} s+a_{6}+a_{7}+a_{10}-\alpha^{\prime}\left(-a_{10} s+\frac{1}{2} a_{12}+a_{14}\right)+\mathcal{O}\left(\alpha^{\prime 2}\right)\right\},
$$

s - channel :

$$
\begin{aligned}
A_{s} & =\frac{i \mathcal{C} \alpha^{\prime 2}}{-4 s}\left\{\frac{1}{2} a_{1} t+a_{5}-a_{6}-a_{7}+a_{8}+a_{9}-a_{10}-\left(\frac{\alpha^{\prime}}{4}\right)\left[\left(a_{4}+a_{7}+a_{8}\right) t\right.\right. \\
& \left.\left.-2 a_{12}+2 a_{13}-4 a_{14}+4 a_{15}\right]+\mathcal{O}\left(\alpha^{\prime 2}\right)\right\}
\end{aligned}
$$

contact - terms :

$$
\begin{aligned}
A_{c} & =\frac{i \mathcal{C} \alpha^{\prime 2}}{2}\left\{-\left(a_{1}+a_{2}-a_{3}\right)+\left(\frac{\alpha^{\prime}}{2}\right)\left[a_{4}+a_{7}+a_{8}-a_{9}+a_{10}\right.\right. \\
& \left.\left.-\left(-2 s-\frac{1}{2} t\right)\left(a_{1}+a_{2}+a_{3}\right)\right]+\mathcal{O}\left(\alpha^{\prime 2}\right)\right\} .
\end{aligned}
$$

\section{Low energy effective action}

In this section we will derive the low energy action for the above amplitude to the first two leading orders of $\alpha^{\prime}$. For scattering of two gravitons we use the physical condition for them as,

$$
\epsilon_{\mu \nu}=\epsilon_{\nu \mu} \quad, \quad \epsilon_{\mu}^{\mu}=0
$$

In the t-channel we have two gravitons which interact in the bulk space and produce another graviton or dilaton field which in turn is absorbed by the D-brane. 
It is easily seen that the massless antisymmetric field does not contribute to the amplitude. The t-channel amplitude to be reproduced by inserting for the coefficients $a_{i}$ of appendix A,

$$
\begin{aligned}
A_{t} & =\frac{i \mathcal{C} \alpha^{\prime 2}}{t}\left\{\left(k_{1} \cdot D_{S} \cdot k_{1}\right) \operatorname{Tr}\left(\epsilon_{1} \cdot \epsilon_{2}\right)+\operatorname{Tr}\left(\epsilon_{1} \cdot D_{S}\right)\left(k_{1} \cdot \epsilon_{2} \cdot k_{1}\right)+\operatorname{Tr}\left(\epsilon_{2} \cdot D_{S}\right)\left(k_{2} \cdot \epsilon_{1} \cdot k_{2}\right)\right. \\
& -2 k_{2} \cdot \epsilon_{1} \cdot D_{S} \cdot \epsilon_{2} \cdot k_{1}-2 k_{1} \cdot \epsilon_{2} \cdot \epsilon_{1} \cdot k_{2}-2 k_{1} \cdot D_{S} \cdot \epsilon_{1} \cdot \epsilon_{2} \cdot k_{1}-2 k_{2} \cdot D_{S} \cdot \epsilon_{2} \cdot \epsilon_{1} \cdot k_{2} \\
& -\alpha^{\prime}\left[\left(k_{1} \cdot \epsilon_{2} \cdot \epsilon_{1} \cdot k_{2}\right)\left(k_{1} \cdot D_{S} \cdot k_{1}\right)+\left(k_{1} \cdot \epsilon_{2} \cdot k_{1}\right)\left(k_{2} \cdot \epsilon_{1} \cdot k_{2}\right)+\left(k_{1} \cdot \epsilon_{2} \cdot k_{1}\right)\left(k_{2} \cdot \epsilon_{1} \cdot D_{S} \cdot k_{2}\right)\right. \\
& \left.\left.+\left(k_{2} \cdot \epsilon_{1} \cdot k_{2}\right)\left(k_{1} \cdot \epsilon_{2} \cdot D_{S} \cdot k_{1}\right)\right]\right\}=A_{t}^{0}+A_{t}^{1},
\end{aligned}
$$

where $A_{t}^{0}\left(A_{t}^{1}\right)$ contains terms in the curly bracket which are zeroth (first) order at $\alpha^{\prime}$. It is not difficult to see that the action which describes this amplitude to order of $\alpha^{\prime 0}$ is nothing but the DBI and Einstein-Hilbert action. The DBI action in the Einstein frame is ${ }^{5}$

$$
\mathcal{S}_{D-\text { brane }}^{0}=-T_{p} \int d^{p+1} x e^{-\Phi} \sqrt{-\operatorname{det}\left(e^{-\gamma \Phi} g+\mathcal{B}+f\right)_{a b}},
$$

where $g$ is the induced metric on the D-brane and $\mathcal{B}_{a b}=B_{a b}-2 \kappa b_{a b}$ is the pull back of the antisymmetric field along the D-brane with $B$ constant and $f_{a b}$ is the gauge field strength on the D-brane and $\gamma=-\frac{4}{d-2}$. Expanding $g_{a b}$ around the Minkowski metric, $g_{a b}=\eta_{a b}+2 \kappa h_{a b}$ we get for the action to the first order of $h,{ }^{6}$

$$
\mathcal{S}_{D-\text { brane }}^{0}=-\kappa T_{p} c \int d^{p+1} x h_{a b} V^{a b},
$$

where

$$
V^{a b}=\frac{1}{2}(\eta+D)^{b a}, \quad c=\sqrt{-\operatorname{det}(\eta+B)_{a b}} .
$$

Equation (28) exhibits a source term for gravity on the D-brane,

$$
\left(S_{h}\right)^{a b}=-\frac{1}{2} T_{p} \kappa c\left(\eta^{a b}+D_{S}^{a b}\right) .
$$

The Einstein-Hilbert action in the bulk is

$$
S_{b u l k}^{(0)}=\frac{1}{2 \kappa^{2}} \int d^{26} x \sqrt{-G} R .
$$

Using 26-dimensional propagator $\left(G^{h h}\right)_{\mu \nu \lambda \rho}$ and the three point interaction vertex $\left(V_{h \epsilon_{1} \epsilon_{2}}\right)^{\lambda \rho}$ for gravitons coming from this action [6], and the above source term, we find

$$
i\left(S_{h}\right)^{\mu \nu}\left(G^{h h}\right)_{\mu \nu \lambda \rho}\left(V_{h \epsilon_{1} \epsilon_{2}}\right)^{\lambda \rho}=A_{t}^{0}+C_{t}^{0},
$$

\footnotetext{
${ }^{5}$ For simplicity in writing we have dropped the coefficient of $f$ i.e. $2 \pi \alpha^{\prime} f \rightarrow f$.

${ }^{6}$ Note that we have expanded the action (27) around the background $\eta_{a b}+B_{a b}$ using $\sqrt{\operatorname{det}\left(M_{0}+M\right)}=\sqrt{\operatorname{det} M_{0}}\left(1+\frac{1}{2} \operatorname{Tr}\left(M_{0}^{-1} M\right)+\ldots\right)$.
} 
where $A_{t}^{0}$ is exactly the zeroth order term of the amplitude (26), and $C_{t}^{0}$ is a contact term with no poles,

$$
C_{t}^{0}=-i c T_{p} \kappa^{2}\left(\operatorname{Tr}\left(\epsilon_{1} \cdot \epsilon_{2}\right)+\operatorname{Tr}\left(\epsilon_{1} \cdot D_{S} \cdot \epsilon_{2}\right)\right) .
$$

Here the tension of the D-branes, $T_{p}$ from (27) is written in terms of the coefficients of (26) i.e.

$$
T_{p}=\frac{\mathcal{C} \alpha^{2}}{c \kappa^{2}} .
$$

We note that to this order of $\alpha^{\prime}$ the above results are exactly the same as in the superstring theory [6]. To get the next order terms in the string amplitude (26), we include the next order of $\alpha^{\prime}$ gravitational action in the bulk, $[3,4]$

$$
S_{b u l k}^{(1)}=\frac{\alpha^{\prime}}{8 \kappa^{2}} \int d^{26} x e^{\gamma \Phi} \sqrt{-G}\left(R^{\mu \nu \kappa \lambda} R_{\mu \nu \kappa \lambda}-4 R^{\mu \nu} R_{\mu \nu}+R^{2}\right) .
$$

There is a new three point interaction for gravitons $V_{h \epsilon_{1} \epsilon_{2}}^{\prime}$ in this Lagrangian. In addition it contains the interaction between one dilaton and two gravitons $V_{\Phi \epsilon_{1} \epsilon_{2}}$. So there are two source terms, gravitational source $S_{h}$ and dilatonic source $S_{\Phi}$ and we find

$$
i S_{h} G^{h h} V_{h \epsilon_{1} \epsilon_{2}}^{\prime}+i S_{\Phi} G^{\Phi \Phi} V_{\Phi \epsilon_{1} \epsilon_{2}}=A_{t}^{1}+C_{t}^{1}
$$

where $A_{t}^{1}$ is the first order term of the amplitude (26) and

$$
\begin{aligned}
C_{t}^{1} & =\frac{i c T_{p} \kappa^{2} \alpha^{\prime}}{2}\left\{-k_{1} \cdot D_{S} \cdot k_{1} \operatorname{Tr}\left(\epsilon_{1} \cdot \epsilon_{2}\right)+k_{1} \cdot k_{2} \operatorname{Tr}\left(\epsilon_{1} \cdot D_{S} \cdot \epsilon_{2}\right)-k_{1} \cdot \epsilon_{2} \cdot \epsilon_{1} \cdot D_{S} \cdot k_{2}\right. \\
& \left.\left.+k_{2} \cdot D_{S} \cdot \epsilon_{2} \cdot \epsilon_{1} \cdot k_{2}-k_{1} \cdot \epsilon_{2} \cdot D_{S} \cdot \epsilon_{1} \cdot k_{2}\right)\right\}
\end{aligned}
$$

is a contact term. This calculation exhibits similar gravitational source term as in the order of $\alpha^{\prime 0}$.

Next we consider the s-channel amplitude up to the first two powers of $\alpha^{\prime}$. We may write this amplitude, (23), as

$$
\begin{aligned}
A_{s} & =\frac{-i c \kappa^{2} T_{p}}{4 s}\left\{\operatorname{Tr}\left(\epsilon_{1} \cdot D_{S}\right)\left(k_{1} \cdot \epsilon_{2} \cdot D_{S} \cdot k_{2}-k_{1} \cdot D_{S} \cdot \epsilon_{2} \cdot D_{S} \cdot k_{2}-k_{1} \cdot D_{A} \cdot \epsilon_{2} \cdot D_{A} \cdot k_{2}\right)\right. \\
& +k_{2} \cdot D_{A} \cdot \epsilon_{2} \cdot D_{S} \cdot \epsilon_{1} \cdot D_{A} \cdot k_{1}+k_{2} \cdot D_{S} \cdot \epsilon_{2} \cdot D_{S} \cdot \epsilon_{1} \cdot D_{S} \cdot k_{1}+2 k_{2} \cdot D_{A} \cdot \epsilon_{2} \cdot D_{A} \cdot \epsilon_{1} \cdot D_{S} \cdot k_{1} \\
& -k_{2} \cdot D_{S} \cdot \epsilon_{2} \cdot \epsilon_{1} \cdot D_{S} \cdot k_{1}+k_{2} \cdot D_{A} \cdot \epsilon_{2} \cdot \epsilon_{1} \cdot D_{A} \cdot k_{1}-\frac{1}{2} k_{1} \cdot k_{2} \operatorname{Tr}\left(\epsilon_{1} \cdot D_{S}\right) \operatorname{Tr}\left(\epsilon_{2} \cdot D_{S}\right) \\
& +\left(\frac{\alpha^{\prime}}{2}\right)\left[k_{1} \cdot k_{2} \operatorname{Tr}\left(\epsilon_{1} \cdot D_{S}\right)\left(k_{2} \cdot D \cdot \epsilon_{2} \cdot D \cdot k_{2}\right)+\left(k_{2} \cdot \epsilon_{1} \cdot D_{S} \cdot k_{1}\right)\left(k_{1} \cdot \epsilon_{2} \cdot D_{S} \cdot k_{2}\right)\right. \\
& -\left(k_{1} \cdot D_{S} \cdot \epsilon_{1} \cdot D_{S} \cdot k_{2}\right)\left(k_{2} \cdot D_{S} \cdot \epsilon_{2} \cdot D_{S} \cdot k_{1}\right)+2\left(k_{1} \cdot D_{A} \cdot \epsilon_{2} \cdot D_{A} \cdot k_{1}\right)\left(k_{1} \cdot D_{S} \cdot \epsilon_{1} \cdot D_{S} \cdot k_{2}\right)
\end{aligned}
$$




$$
\left.\left.-\left(k_{1} \cdot D_{A} \cdot \epsilon_{2} \cdot D_{A} \cdot k_{1}\right)\left(k_{2} \cdot D_{A} \cdot \epsilon_{1} \cdot D_{A} \cdot k_{2}\right)\right]\right\}+(1 \leftrightarrow 2)=A_{s}^{0}+A_{s}^{1}
$$

In this channel we expect the two gravitons scatter from the D-brane and exchange a gauge or scalar field which propagates on the D-brane. These propagating fields are in fact the low energy limits of the massless open strings on the D-brane, scalars $\left(X^{i}\right)$ and gauge bosons $\left(a_{a}\right)$. We would like to write down a Lagrangian for these fields on the D-brane which produces the vertices for interactions between gravitons and these fields and finally gives the scattering amplitude of the string theory. For zeroth order of $\alpha^{\prime}$ we again take the DBI action on D-brane (27) with gauge field strength defined as $f_{a b}=\partial_{a} a_{b}-\partial_{b} a_{a}$ then expand the induced metric $g_{a b}=\partial_{a} X^{\mu} \partial_{b} X^{\nu} g_{\mu \nu}$ on the D-brane around the flat space and choose the static gauge $\left(X^{a}=x^{a}\right)$ to get

$$
g_{a b}=\eta_{a b}+2 \kappa h_{a b}+2 \kappa\left(h_{i a} \partial_{b} X^{i}+h_{b i} \partial_{a} X^{i}\right)+\partial_{a} X^{i} \partial_{b} X_{i}+2 \kappa h_{i j} \partial_{a} X^{i} \partial_{b} X^{j} .
$$

Redefining the gauge and scalar fields by $A_{a}=2 \pi \alpha^{\prime} \sqrt{T_{p}} a_{a}$ and $\lambda^{i}=\sqrt{T_{p}} X^{i}$ and expanding DBI action around the background field $\eta_{a b}+B_{a b}$, for the zeroth order of $\alpha^{\prime}$ we find the following vertices,

$$
\begin{aligned}
& \left(V_{\epsilon_{1} A}\right)^{a}=\sqrt{T_{p}} \kappa c\left(\operatorname{Tr}\left(\epsilon_{1} \cdot V_{S}\right) k_{1} \cdot V_{A}^{a}-2 k_{1} \cdot V_{S} \cdot \epsilon_{1} \cdot V_{A}^{a}-2 k_{1} \cdot V_{A} \cdot \epsilon_{1} \cdot V_{S}^{a}\right), \\
& \left(V_{\epsilon_{1} \lambda}\right)_{i}=\sqrt{T_{p}} \kappa c\left(\operatorname{Tr}\left(\epsilon_{1} \cdot V_{S}\right) k_{1 i}-2 k_{1} \cdot V_{S} \cdot \epsilon_{1 i}\right)
\end{aligned}
$$

and propagators,

$$
\left(G^{\lambda \lambda}\right)^{i j}=\frac{i}{c s} \eta^{i j} \quad, \quad\left(G^{A A}\right)_{a b}=\frac{i}{c s}\left(V_{S}^{-1}\right)_{a b}
$$

where $V_{A}$ and $V_{S}$ are the antisymmetric and symmetric parts of the matrix $V$. Note that in deriving the above vertices we have used the fact that the closed string fields in the DBI action appear as functionals of the transverse scalar fields $X^{i}$ which should be Taylor expanded. Knowing these vertices and propagators we can compute the amplitude from the effective field theory on D-brane and show that to order of $\alpha^{\prime 0}$,

$$
V_{\epsilon_{1} \lambda} G^{\lambda \lambda} V_{\lambda \epsilon_{2}}+V_{\epsilon_{1} A} G^{A A} V_{A \epsilon_{2}}=A_{s}^{0}+C_{s}^{0},
$$

where $A_{s}^{0}$ is the zeroth order term in (38) and $C_{s}^{0}$ is a contact term,

$$
C_{s}^{0}=\frac{-i \kappa^{2} T_{p} c}{4} \operatorname{Tr}\left(\epsilon_{1} \cdot D_{S}\right) \operatorname{Tr}\left(\epsilon_{2} \cdot D_{S}\right)
$$

We have used the following useful relations in the derivation

$$
\left(V_{A} V_{S}^{-1} V_{A}\right)^{a b}=-\frac{1}{2}\left(\eta-D_{S}\right)^{a b} \quad, \quad \eta^{i j}=\frac{1}{2}\left(\eta-D_{S}\right)^{i j}
$$

It turns out that in order to describe the amplitude to the first order in $\alpha^{\prime}$ we need to add new terms to the DBI action as follow 


$$
\begin{aligned}
\mathcal{S}^{1} & =\frac{-\alpha^{\prime} T_{p}}{2} \int d^{p+1} x\left\{\sqrt{-\operatorname{det}(\eta+B+f)} R_{a b c d}\left(\frac{1}{\eta+B+f}\right)^{a d}\left(\frac{1}{g+B+f}\right)^{b c}\right. \\
& \left.-\sqrt{-\operatorname{det}(g+B+f)}\left(\Omega_{a c}^{i} \Omega_{i b d}-\Omega_{a d}^{i} \Omega_{i b c}\right)\left(\frac{1}{g+B+f}\right)^{a d}\left(\frac{1}{g+B+f}\right)^{b c}\right\},
\end{aligned}
$$

where $R_{a b c d}=g_{a e} R_{b c d}^{e}$ and $R_{b c d}^{e}$ is Riemann tensor constructed out of induced metric on the D-brane and $\Omega$ 's are second fundamental forms,

$$
\begin{aligned}
R_{b c d}^{a} & =\Gamma_{b d, c}^{a}-\Gamma_{b c, d}^{a}+\Gamma_{b d}^{e} \Gamma_{c e}^{a}-\Gamma_{b c}^{e} \Gamma_{d e}^{a}, \\
\Omega_{a b}^{i} & =\kappa\left(-\partial^{i} h_{a b}+\partial_{a} h_{b}^{i}+\partial_{b} h_{a}^{i}\right)+\partial_{a} \partial_{b} X^{i},
\end{aligned}
$$

which $a, b, c, d, e=0, \ldots, p$ and $i, j=p+1, \ldots d$. The $\Gamma$ 's are the Christoffel symbols constructed out of the induced metric $g$. This action contains every vertex that we need for producing the next order of $\alpha^{\prime}$ in (38), in addition to certain contact terms which we will describe later. To reproduce the s-channel amplitude of string theory from such an action we need to find the graviton-scalar and graviton-gauge field vertices. We find the first one from the second fundamental form linear in terms of $X$ and $h,{ }^{7}$ and the second one from the expansion the above Lagrangian linear in terms of $f$ and $h$. By summing these vertices corresponding to the above action with the previous terms which we found from DBI action to zeroth order of $\alpha^{\prime}$, we find that

$$
\begin{aligned}
\left(V_{\epsilon_{1} A}\right)^{a} & =\frac{\kappa c \sqrt{T_{p}}}{4}\left\{2 \operatorname{Tr}\left(\epsilon_{1} \cdot D_{S}\right) k_{1} \cdot V_{A}^{a}-4 k_{1} \cdot D_{S} \cdot \epsilon_{1} \cdot V_{A}^{a}+4 k_{1} \cdot D_{A} \cdot \epsilon_{1} \cdot V_{S}^{a}\right. \\
& \left.-\alpha^{\prime}\left[\left(k_{1} \cdot D_{S} \cdot \epsilon_{1} \cdot D_{S} \cdot k_{1}+k_{1} \cdot D_{A} \cdot \epsilon_{1} \cdot D_{A} \cdot k_{1}\right) k_{1} \cdot V_{A}^{a}-k_{1} \cdot D_{S} \cdot k_{1} \operatorname{Tr}\left(\epsilon_{1} \cdot D_{S}\right) k_{1} \cdot V_{A}^{a}\right]\right\} \\
\left(V_{\epsilon_{1} \lambda}\right)_{i} & =\frac{\kappa c \sqrt{T_{p}}}{4}\left\{2 \operatorname{Tr}\left(\epsilon_{1} \cdot D_{S}\right) k_{1 i}-4 k_{1} \cdot D_{S} \cdot \epsilon_{1 i}\right. \\
& \left.-\alpha^{\prime}\left[\left(k_{1} \cdot D_{S} \cdot \epsilon_{1} \cdot D_{S} \cdot k_{1}+k_{1} \cdot D_{A} \cdot \epsilon_{1} \cdot D_{A} \cdot k_{1}\right) k_{1 i}-k_{1} \cdot D_{S} \cdot k_{1} \operatorname{Tr}\left(\epsilon_{1} \cdot D_{S}\right) k_{1 i}\right]\right\} .
\end{aligned}
$$

To check that these vertices are in fact correct we have calculated one closed and one open string scattering from D-brane and we have found similar vertices (see Appendix C). Now using these vertices we can find the first order of $\alpha^{\prime}$ terms in s-channel from string theory. We have used the previous propagators for gauge and scalar fields, as (45) does not introduce any corrections to the propagators. We then find

$$
V_{\epsilon_{1} \lambda} G^{\lambda \lambda} V_{\lambda \epsilon_{2}}+V_{\epsilon_{1} A} G^{A A} V_{A \epsilon_{2}}=A_{s}^{0}+C_{s}^{0}+A_{s}^{1}+C_{s}^{1},
$$

\footnotetext{
${ }^{7}$ We must Taylor expand the linear terms in $h$ of the above action, but because of momentum conservation relation these terms do not contribute to the vertex.
} 
in which $A_{s}^{0}\left(A_{s}^{1}\right)$ is exactly the zeroth (first) order of $\alpha^{\prime}$ terms of (38), $C_{s}^{0}$ is the zeroth order term in (43) and $C_{s}^{1}$ is the first order in $\alpha^{\prime}$ terms with no poles and can be written as

$$
\begin{aligned}
C_{s}^{1} & =\frac{i c \kappa^{2} T_{p} \alpha^{\prime}}{4}\left\{\frac{1}{2} \operatorname{Tr}\left(\epsilon_{1} \cdot D_{S}\right) \operatorname{Tr}\left(\epsilon_{2} \cdot D_{S}\right)\left(k_{1} \cdot D_{S} \cdot k_{2}-k_{1} \cdot k_{2}\right)+\right. \\
& \left.+\operatorname{Tr}\left(\epsilon_{1} \cdot D_{S}\right)\left[2 k_{2} \cdot D_{S} \cdot \epsilon_{2} \cdot k_{1}+\frac{3}{2} k_{2} \cdot D \cdot \epsilon_{2} \cdot D \cdot k_{2}\right]\right\}+(1 \leftrightarrow 2) .
\end{aligned}
$$

It remains to verify that all the above contact terms (33), (43) and contact terms from (27) at $\alpha^{\prime 0}$ and (37), (49) and contact terms from (45) at $\alpha^{\prime}$ add up to the contact terms of the string amplitude (24). We can write (24) as

$$
\begin{aligned}
A_{c} & =\frac{i c \kappa^{2} T_{p}}{4}\left\{-\operatorname{Tr}\left(\epsilon_{1} \cdot D\right) \operatorname{Tr}\left(\epsilon_{2} \cdot D\right)-\operatorname{Tr}\left(\epsilon_{1} \cdot \epsilon_{2}\right)+\operatorname{Tr}\left(D \cdot \epsilon_{1} \cdot D \cdot \epsilon_{2}\right)\right. \\
& +\alpha^{\prime}\left[\operatorname{Tr}\left(\epsilon_{1} \cdot D\right)\left(k_{2} \cdot D \cdot \epsilon_{2} \cdot D \cdot k_{2}\right)-k_{1} \cdot D \cdot \epsilon_{2} \cdot D \cdot \epsilon_{1} \cdot D \cdot k_{2}+k_{1} \cdot \epsilon_{2} \cdot \epsilon_{1} \cdot k_{2}\right. \\
& \left.+\frac{1}{2}\left(k_{1} \cdot D \cdot k_{2}\right)\left(\operatorname{Tr}\left(\epsilon_{1} \cdot D\right) \operatorname{Tr}\left(\epsilon_{2} \cdot D\right)+\operatorname{Tr}\left(\epsilon_{1} \cdot \epsilon_{2}\right)+\operatorname{Tr}\left(D \cdot \epsilon_{1} \cdot D \cdot \epsilon_{2}\right)\right]\right\} \\
& +(1 \longleftrightarrow 2) .
\end{aligned}
$$

If we expand DBI action (27), we find graviton-graviton contact terms from this expansion,

$$
A_{h h}^{0}=-2 i \kappa^{2} c T_{p}\left\{\frac{1}{2} \operatorname{Tr}\left(\epsilon_{1} \cdot V\right) \operatorname{Tr}\left(\epsilon_{2} \cdot V\right)-\operatorname{Tr}\left(\epsilon_{1} \cdot V \cdot \epsilon_{2} \cdot V\right)\right\}
$$

We can show that by adding (33) and (43) to the above equation

$$
A_{h h}^{0}+C_{t}^{0}+C_{s}^{0}=\frac{-i \kappa^{2} c T_{p}}{2}\left\{\operatorname{Tr}\left(\epsilon_{1} . D\right) \operatorname{Tr}\left(\epsilon_{2} . D\right)+\operatorname{Tr}\left(\epsilon_{1} . \epsilon_{2}\right)-\operatorname{Tr}\left(\epsilon_{1} . D . \epsilon_{2} . D\right)\right\}
$$

we find exactly the $\alpha^{\prime 0}$ term in (50). Again as before if we expand Lagrangian in (45), we find for graviton-graviton contact terms,

$$
\begin{aligned}
A_{h h}^{1} & =-2 i \alpha^{\prime} \kappa^{2} c T_{p}\left\{-k_{1} \cdot V \cdot \epsilon_{2} \cdot \epsilon_{1} \cdot V \cdot k_{2}-k_{1} \cdot V \cdot \epsilon_{2} \cdot V \cdot \epsilon_{1} \cdot k_{2}-k_{1} \cdot \epsilon_{2} \cdot V \cdot \epsilon_{1} \cdot V \cdot k_{2}\right. \\
& +k_{1} \cdot V \cdot \epsilon_{2} \cdot V \cdot \epsilon_{1} \cdot V \cdot k_{2}-\frac{1}{4} k_{1} \cdot k_{2} \operatorname{Tr}\left(\epsilon_{1} \cdot V\right) \operatorname{Tr}\left(\epsilon_{2} \cdot V\right)+\frac{1}{2} k_{1} \cdot V \cdot k_{2} \operatorname{Tr}\left(\epsilon_{1} \cdot V \cdot \epsilon_{2}\right) \\
& +\frac{1}{4} k_{1} \cdot k_{2} \operatorname{Tr}\left(V \cdot \epsilon_{1} \cdot V \cdot \epsilon_{2}\right)+\frac{1}{2} k_{1} \cdot V \cdot k_{1} \operatorname{Tr}\left(V \cdot \epsilon_{1} \cdot V \cdot \epsilon_{2}\right) \\
& \left.+\left(k_{1} \cdot V \cdot \epsilon_{1} \cdot k_{2}+\frac{1}{2} k_{1} \cdot V \cdot \epsilon_{1} \cdot V \cdot k_{1}\right) \operatorname{Tr}\left(\epsilon_{2} \cdot V\right)\right\}+(1 \leftrightarrow 2),
\end{aligned}
$$

we can show that by adding (37) and (49) to above equation, 


$$
\begin{aligned}
A_{h h}^{1}+C_{t}^{1}+C_{s}^{1} & =\frac{i \alpha^{\prime} \kappa^{2} c T_{p}}{4}\left\{\operatorname{Tr}\left(\epsilon_{1} \cdot D\right) k_{2} \cdot D \cdot \epsilon_{2} \cdot D \cdot k_{2}-k_{1} \cdot D \cdot \epsilon_{2} \cdot D \cdot \epsilon_{1} \cdot D \cdot k_{2}+k_{1} \cdot \epsilon_{2} \cdot \epsilon_{1} \cdot k_{2}\right. \\
& \left.+\frac{1}{2}\left(k_{1} \cdot D \cdot k_{2}\right)\left[\operatorname{Tr}\left(\epsilon_{1} \cdot D\right) \operatorname{Tr}\left(\epsilon_{2} \cdot D\right)+\operatorname{Tr}\left(\epsilon_{1} \cdot \epsilon_{2}\right)+\operatorname{Tr}\left(D \cdot \epsilon_{1} \cdot D \cdot \epsilon_{2}\right)\right]\right\} \\
& +(1 \longleftrightarrow 2),
\end{aligned}
$$

we recover exactly the $\alpha^{\prime}$ term in (50). It is noticeable that in evaluating the tchannel poles in field theory we use the linear coupling of graviton to the D-brane using the DBI action (27) which is of zero order of $\alpha^{\prime}$ whereas linear coupling of graviton to D-brane coming from action (45) is of first order of $\alpha^{\prime}$ however using conservation of momentum this coupling has no effect in the t-channel.

It should be noted that when we turn off the $B$ field on the D-brane we find the Einstein-Hilbert action as we expected [14]. To see this, note that as we turn off the $B$ field on the D-brane we see, $V^{a b}=\eta^{a b}$ where upon (45) changes up to some total derivatives to

$$
\begin{aligned}
\mathcal{S}_{D-\text { brane }}^{1} & =-\frac{\alpha^{\prime} T_{p}}{2} \int d^{p+1} x \sqrt{-\operatorname{det}_{a b}}\left(R+\Omega^{i}{ }_{a}^{a} \Omega_{i b}{ }^{b}-\Omega^{i}{ }_{a b} \Omega_{i}{ }^{a b}\right), \\
R & =R_{a b c d} g^{a c} g^{b d}
\end{aligned}
$$

which $a, b, c, d=0, \ldots, p$ and $i, j=p+1, \ldots d$. Where we have ignored gauge fields because they do not have any contraction with gravitons because of the antisymmetry of $f_{a b}$. In deriving the above equation we have used the following relation at $\mathcal{O}\left(h^{2}\right)$

$$
\sqrt{-\operatorname{det} \eta} R_{a b c d} \eta^{a d} g^{b c}=\sqrt{-\operatorname{det} g} R_{a b c d} g^{a c} g^{b d}+(T . D)
$$

which by momentum conservation relation, total derivative terms have no effect in scattering amplitude.

\section{Discussion}

In this work we have found the corrections to the DBI action to order of $\alpha^{\prime},(45)$, for the description of the graviton- graviton-D-brane scattering in bosonic string theory in the presence of a constant $B$ field.

We have also calculated the corrections to the DBI action for dilaton and antisymmetric field scattering with a D-brane. The results are not qualitatively different and will be reported separately.

It is known that D-branes in the presence of background $B$ field become noncommutative and the DBI action can be written in terms of commutative gauge fields or in terms of the noncommutative open string fields involve the $\star$ product and a Wilson line operator. We write the action (45) in terms of commutative fields.

One may write the result in eq(45) in the following form, 


$$
\begin{aligned}
\mathcal{S}^{1} & =\frac{-\alpha^{\prime} T_{p}}{2} \int d^{p+1} x \sqrt{-\operatorname{det}(\eta+B+f)}\left(\frac{1}{\eta+B+f}\right)^{a d}\left(\frac{1}{g+B+f}\right)^{b c} \\
& \times\left\{R_{a b c d}-\Omega^{i}{ }_{a c} \Omega_{i b d}+\Omega^{i}{ }_{a d} \Omega_{i b c}\right\}
\end{aligned}
$$

which is reminiscent of the corresponding result for the case $B=0$, eq (55). This action as well as the action in (45) are not in covariant form. One may try to write it in a covariant form;

$$
\begin{aligned}
\mathcal{S}_{\text {cov }}^{1} & =\frac{-\alpha^{\prime} T_{p}}{2} \int d^{p+1} x \sqrt{-\operatorname{det}(g+B+f)}\left(\frac{1}{g+B+f}\right)^{a d}\left(\frac{1}{g+B+f}\right)^{b c} \\
& \times\left\{R_{a b c d}-\Omega^{i}{ }_{a c} \Omega_{i b d}+\Omega^{i}{ }_{a d} \Omega_{i b c}\right\}
\end{aligned}
$$

but, this will give rise to new contact terms which are not present in the string theory amplitude, eq. (50). However, on general grounds one expects a covariant form for the action. It is conceivable that addition of terms involving covariant derivatives of the gauge field $\mathrm{f}$ contracted with the metric $\mathrm{g}$ or $\mathrm{g}+\mathrm{B}$ may conspire to cancel these unwanted terms and reproduce the string amplitude.

In view of the large variety of such possible terms and the complication of these calculations, we have not been able to find a simple covariant variation of the action (57). The same difficulties arise for application of $\mathrm{T}$ duality on the action which would be a check of our result.

\section{Acknowledgments}

We would like to thank M. Alishahiha and S. F. Hassan for useful conversations. F.A acknowledges discussion with L. Alvarez-Gaume. 


\section{Appendix A}

\section{Coefficients for Noncommutative D-brane}

Here we presented the coefficient for scattering of two closed string from D-brane in the presence of $B$ field.

$$
\begin{aligned}
a_{1} & =\operatorname{Tr}\left(\epsilon_{1} \cdot D\right) \operatorname{Tr}\left(\epsilon_{2} \cdot D\right) \\
a_{2} & =\operatorname{Tr}\left(\epsilon_{1}^{T} \cdot \epsilon_{2}\right) \\
a_{3} & =\operatorname{Tr}\left(D \cdot \epsilon_{1} \cdot D \cdot \epsilon_{2}\right) \\
a_{4} & =\operatorname{Tr}\left(\epsilon_{1} \cdot D\right)\left(k_{1} \cdot \epsilon_{2} \cdot D \cdot k_{1}+k_{1} \cdot D \cdot \epsilon_{2} \cdot k_{1}\right)+(1 \longleftrightarrow 2) \\
a_{5} & =k_{1} \cdot \epsilon_{2} \cdot D \cdot \epsilon_{1} D \cdot k_{2}+k_{2} \cdot \epsilon_{1} \cdot D \cdot \epsilon_{2} \cdot D \cdot k_{1}+k_{1} \cdot D \cdot \epsilon_{2} \cdot D \cdot \epsilon_{1} \cdot k_{2}+k_{2} \cdot D \cdot \epsilon_{1} \cdot D \cdot \epsilon_{2} \cdot k_{1} \\
& -k_{2} \cdot D^{T} \cdot \epsilon_{1}^{T} \cdot \epsilon_{2} \cdot D \cdot k_{1}-k_{2} \cdot D \cdot \epsilon_{1} \cdot \epsilon_{2}^{T} \cdot D^{T} \cdot k_{1} \\
a_{6} & =k_{2} \cdot D^{T} \cdot \epsilon_{1}^{T} \cdot \epsilon_{2} \cdot k_{1}+k_{2} \cdot \epsilon_{1}^{T} \cdot \epsilon_{2} \cdot D \cdot k_{1}-k_{1} \cdot \epsilon_{2} \cdot D \cdot \epsilon_{1} \cdot k_{2}-k_{2} \cdot \epsilon_{1} \cdot D \cdot \epsilon_{2} \cdot k_{1} \\
& +k_{2} \cdot \epsilon_{1} \cdot \epsilon_{2}^{T} \cdot D^{T} \cdot k_{1}+k_{2} \cdot D \cdot \epsilon_{1} \cdot \epsilon_{2}^{T} \cdot k_{1} \\
a_{7} & =\operatorname{Tr}\left(\epsilon_{1} \cdot D\right)\left(k_{1} \cdot \epsilon_{2} \cdot k_{1}\right)+(1 \longleftrightarrow 2) \\
a_{15} & =\left(k_{1} \cdot D \cdot \epsilon_{2} \cdot D \cdot k_{1}\right)\left(k_{2} \cdot D \cdot \epsilon_{1} \cdot D \cdot k_{2}\right) \cdot \\
a_{14} & =\left(k_{1} \cdot \epsilon_{2} \cdot k_{1}\right)\left(k_{2} \cdot \epsilon_{1} \cdot k_{2}\right) \\
a_{8} & =\operatorname{Tr}\left(\epsilon_{1} \cdot D\right)\left(k_{1} \cdot D \cdot \epsilon_{2} \cdot D \cdot k_{1}\right)+(1 \longleftrightarrow 2) \\
a_{12} & =\left(k_{1} \cdot \epsilon_{2} \cdot k_{1}\right)\left(k_{2} \cdot \epsilon_{1} \cdot D \cdot k_{2}+k_{2} \cdot D \cdot \epsilon_{1} \cdot k_{2}\right)+(1 \longleftrightarrow 2) \\
a_{9} & =k_{1} \cdot D \cdot \epsilon_{2} \cdot D \cdot \epsilon_{1} \cdot D \cdot k_{2}+(1 \longleftrightarrow 2) \\
a_{10} & =k_{2} \cdot \epsilon_{1}^{T} \cdot \epsilon_{2} \cdot k_{1}+k_{2} \cdot \epsilon_{1} \cdot \epsilon_{2}^{T} \cdot k_{1} \\
a_{11} & =\left(k_{1} \cdot \epsilon_{2} \cdot D \cdot k_{1}+k_{1} \cdot D \cdot \epsilon_{2} \cdot k_{1}\right)\left(k_{2} \cdot \epsilon_{1} \cdot D \cdot k_{2}+k_{2} \cdot D \cdot \epsilon_{1} \cdot k_{2}\right) \\
& =\left(k_{2} \cdot D \cdot \epsilon_{1} \cdot D \cdot k_{2}\right)+\left(k_{1} \cdot D \cdot \epsilon_{2} \cdot D \cdot k_{1}\right)\left(k_{2} \cdot \epsilon_{1} \cdot k_{2}\right)
\end{aligned}
$$




\section{Appendix B}

\section{Coefficients for commutative D-brane}

When we turn the $B$ field off we must only change $D_{S}^{a b}=D_{0}^{a b}=\eta^{a b}$ and $D_{S}^{i j}=$ $D_{0}^{i j}=-\delta^{i j}$ and $D_{A}=0$ again we find the same amplitude as (19) by including these changes and replacing $a_{n}$ coefficients with $b_{n}$, where

$$
\begin{aligned}
& b_{1}=\operatorname{Tr}\left(\epsilon_{1} \cdot D_{0}\right) \operatorname{Tr}\left(\epsilon_{2} \cdot D_{0}\right) \\
& b_{2}=\operatorname{Tr}\left(\epsilon_{1}^{T} \cdot \epsilon_{2}\right) \\
& b_{3}=\operatorname{Tr}\left(D_{0} \cdot \epsilon_{1} \cdot D_{0} \cdot \epsilon_{2}\right) \\
& b_{4}=\operatorname{Tr}\left(\epsilon_{1} \cdot D_{0}\right)\left(k_{1} \cdot \epsilon_{2} \cdot D_{0} \cdot k_{1}+k_{1} \cdot D_{0} \cdot \epsilon_{2} \cdot k_{1}\right)+(1 \longleftrightarrow 2) \\
& b_{5}=k_{1} \cdot \epsilon_{2} \cdot D_{0} \cdot \epsilon_{1} \cdot D_{0} \cdot k_{2}+k_{2} \cdot \epsilon_{1} \cdot D_{0} \cdot \epsilon_{2} \cdot D_{0} \cdot k_{1}+k_{1} \cdot D_{0} \cdot \epsilon_{2} \cdot D_{0} \cdot \epsilon_{1} \cdot k_{2}+k_{2} \cdot D_{0} \cdot \epsilon_{1} \cdot D_{0} \cdot \epsilon_{2} \cdot k_{1} \\
& -k_{2} \cdot D_{0} \cdot \epsilon_{1}^{T} \cdot \epsilon_{2} \cdot D_{0} \cdot k_{1}-k_{2} \cdot D_{0} \cdot \epsilon_{1} \cdot \epsilon_{2}^{T} \cdot D_{0} \cdot k_{1} \\
& b_{6}=k_{2} \cdot D_{0} \cdot \epsilon_{1}^{T} \cdot \epsilon_{2} \cdot k_{1}+k_{2} \cdot \epsilon_{1}^{T} \cdot \epsilon_{2} \cdot D_{0} \cdot k_{1}-k_{1} \cdot \epsilon_{2} \cdot D_{0} \cdot \epsilon_{1} \cdot k_{2}-k_{2} \cdot \epsilon_{1} \cdot D_{0} \cdot \epsilon_{2} \cdot k_{1} \\
& +k_{2} \cdot \epsilon_{1} \cdot \epsilon_{2}^{T} \cdot D_{0} \cdot k_{1}+k_{2} \cdot D_{0} \cdot \epsilon_{1} \cdot \epsilon_{2}^{T} \cdot k_{1} \\
& b_{7}=\operatorname{Tr}\left(\epsilon_{1} \cdot D_{0}\right)\left(k_{1} \cdot \epsilon_{2} \cdot k_{1}\right)+(1 \longleftrightarrow 2) \\
& b_{8}=\operatorname{Tr}\left(\epsilon_{1} \cdot D_{0}\right)\left(k_{1} \cdot D_{0} \cdot \epsilon_{2} \cdot D_{0} \cdot k_{1}\right)+(1 \longleftrightarrow 2) \\
& b_{9}=k_{1} \cdot D_{0} \cdot \epsilon_{2} \cdot D_{0} \cdot \epsilon_{1} \cdot D_{0} \cdot k_{2}+(1 \longleftrightarrow 2) \\
& b_{10}=k_{2} \cdot \epsilon_{1}^{T} \cdot \epsilon_{2} \cdot k_{1}+k_{2} \cdot \epsilon_{1} \cdot \epsilon_{2}^{T} \cdot k_{1} \\
& b_{11}=\left(k_{1} \cdot \epsilon_{2} \cdot D_{0} \cdot k_{1}+k_{1} \cdot D_{0} \cdot \epsilon_{2} \cdot k_{1}\right)\left(k_{2} \cdot \epsilon_{1} \cdot D_{0} \cdot k_{2}+k_{2} \cdot D_{0} \cdot \epsilon_{1} \cdot k_{2}\right) \\
& +\left(k_{1} \cdot \epsilon_{2} \cdot k_{1}\right)\left(k_{2} \cdot D_{0} \cdot \epsilon_{1} \cdot D_{0} \cdot k_{2}\right)+\left(k_{1} \cdot D_{0} \cdot \epsilon_{2} \cdot D_{0} \cdot k_{1}\right)\left(k_{2} \cdot \epsilon_{1} \cdot k_{2}\right) \\
& b_{12}=\left(k_{1} \cdot \epsilon_{2} \cdot k_{1}\right)\left(k_{2} \cdot \epsilon_{1} \cdot D_{0} \cdot k_{2}+k_{2} \cdot D_{0} \cdot \epsilon_{1} \cdot k_{2}\right)+(1 \longleftrightarrow 2) \\
& b_{13}=\left(k_{1} \cdot D_{0} \cdot \epsilon_{2} \cdot D_{0} \cdot k_{1}\right)\left(k_{2} \cdot \epsilon_{1} \cdot D_{0} \cdot k_{2}+k_{2} \cdot D_{0} \cdot \epsilon_{1} \cdot k_{2}\right)+(1 \longleftrightarrow 2) \\
& b_{14}=\left(k_{1} \cdot \epsilon_{2} \cdot k_{1}\right)\left(k_{2} \cdot \epsilon_{1} \cdot k_{2}\right) \\
& b_{15}=\left(k_{1} \cdot D_{0} \cdot \epsilon_{2} \cdot D_{0} \cdot k_{1}\right)\left(k_{2} \cdot D_{0} \cdot \epsilon_{1} \cdot D_{0} \cdot k_{2}\right) .
\end{aligned}
$$




\section{Appendix C}

\section{One open and one closed bosonic string scattering}

In this appendix we want to calculate scattering of one massless open string with one massless closed graviton string. In the bosonic case vertices for open and closed string are

$$
\begin{aligned}
& V_{c}=\epsilon_{\mu \lambda} D_{\nu}^{\lambda}: \partial X^{\mu}\left(z_{1}\right) e^{i k_{1} \cdot X\left(z_{1}\right)}:: \bar{\partial} X^{\nu}\left(\bar{z}_{1}\right) e^{i k_{1} \cdot D \cdot X\left(\bar{z}_{1}\right)}:, \\
& V_{o}=\xi_{\alpha}\left(V^{T}\right)_{\rho}^{\alpha}: \partial X^{\rho}(y) e^{2 i k_{2} \cdot V^{T} \cdot X(y)}:
\end{aligned}
$$

where again we have used doubling trick for writing them. For momentum conservation relation we have

$$
k_{1}^{\mu}+k_{1} \cdot D^{\mu}+2 k_{2} \cdot V^{T \mu}=0,
$$

and physical conditions for momentum and polarizations are

$$
\begin{gathered}
k_{1} \cdot \epsilon^{\mu}=0, \quad k_{1}^{2}=0 \quad, \quad \epsilon_{\mu}^{\mu}=0 \\
k_{2} \cdot V^{T} \cdot \xi=0, \quad m_{\text {open }}^{2}=-\left(2 k_{2} \cdot V^{T}\right)^{2}=0 .
\end{gathered}
$$

By using these two last equations and $V^{T} V=\frac{1}{2}\left(\eta+D_{S}\right)$ we find

$$
k_{1} \cdot D_{S} \cdot k_{1}=-\frac{1}{2} m_{\text {open }}^{2}=0 .
$$

By fixing $\mathrm{SL}(2, \mathrm{R})$ invariant we find for amplitude of open-closed scattering from D-brane

$$
\begin{aligned}
& A_{O C D}=\left.g_{c} g_{o} e^{-\lambda} \int d^{2} z_{1} d y\left\langle V_{c} V_{o}\right\rangle\right|_{z=i, y=0} \\
& d^{2} z_{1} d y \rightarrow\left(z_{1}-y\right)\left(\bar{z}_{1}-y\right)\left(z_{1}-\bar{z}_{1}\right),
\end{aligned}
$$

with $(D . V)^{a b}=\left(V^{T}\right)^{a b}$ and $(D . V)^{i j}=-\eta^{i j}$ after a little computation we find graviton-gauge field and graviton-scalar scattering. From this amplitude we read vertices as

$$
\begin{aligned}
\left(V_{\epsilon_{1} A}\right)^{a} & \sim \frac{\kappa c \sqrt{T_{p}}}{4}\left\{2 \operatorname{Tr}\left(\epsilon_{1} \cdot D_{S}\right) k_{1} \cdot V_{A}^{a}-4 k_{1} \cdot D_{S} \cdot \epsilon_{1} \cdot V_{A}^{a}+4 k_{1} \cdot D_{A} \cdot \epsilon_{1} \cdot V_{S}^{a}\right. \\
& \left.-\alpha^{\prime}\left(k_{1} \cdot D_{S} \cdot \epsilon_{1} \cdot D_{S} \cdot k_{1}+k_{1} \cdot D_{A} \cdot \epsilon_{1} \cdot D_{A} \cdot k_{1}\right) k_{1} \cdot V_{A}^{a}\right\} \\
\left(V_{\epsilon_{1} \lambda}\right)_{i} & \sim \frac{\kappa c \sqrt{T_{p}}}{4}\left\{2 \operatorname{Tr}\left(\epsilon_{1} \cdot D_{S}\right) k_{1 i}-4 k_{1} \cdot D_{S} \cdot \epsilon_{1 i}\right. \\
& \left.-\alpha^{\prime}\left(k_{1} \cdot D_{S} \cdot \epsilon_{1} \cdot D_{S} \cdot k_{1}+k_{1} \cdot D_{A} \cdot \epsilon_{1} \cdot D_{A} \cdot k_{1}\right) k_{1 i}\right\}
\end{aligned}
$$


which are exactly the same as (47) if one uses the on shell condition for the states in (47). The last terms in the vertices in (47) vanish upon using the massless condition for open strings, i.e., (64).

Note that the open string vertex operator in our calculation corresponds to noncommutative fields whereas we write the effective action in terms of commutative fields. The difference between them is the Siberg-Witten map. However for the linear open string field there is no difference between commutative and noncommutative fields. 


\section{References}

[1] A. Neveu, J. Scherk, Nucl. Phys. B 36 (1972) 155.

[2] J. Scherk, J. schwartz, Nucl. Phys. B 81 (1974) 118.

[3] B. Zwiebach, Curvature squared terms and string theories, Phys. Lett. B 156 (1985) 315.

[4] R. R. Metsaev, A. A. Tseytlin, Curvature cubed terms in string theory effective actions. Phys. Lett. B 185 (1987) 52.

[5] M.R. Garousi and R.C. Myers, Superstring scattering from D-branes, Nucl. Phys. B 475 (1996) 193-224, preprint hep-th/9603194.

[6] M.R. Garousi , Superstring scattering from D-branes bound states,JHEP 9812 (1998) 008, preprint hep-th/9805078 .

[7] M.R. Garousi, R.C. Myers, World-valume interactions on D-branes, Nucl. Phys. B 542 (1999) 73-88, preprint hep-th/9809100.

[8] A. Hashimoto and I.R. Klebanov, Scattering of strings from D-branes, Nucl. Phys. Proc. Suppl. 55 B (1997) 118-133, preprint hep-th/9611214 .

[9] C.P. Bachas, P. Bain, M.B. Green, Curvature terms in D-brane actions and their M-theory origin, JHEP 9905 (1999) 011, preprint hep-th/9903210.

[10] A. Fotopoulos, On $\left(\alpha^{\prime}\right)^{2}$ corrections to the D-brane action for non-geodesic world-volume embeddings, JHEP 0109 (2001) 005, preprint hep-th/0104146.

[11] F. Ardalan, H. Arfaei, and M.M. Sheikh-Jabbari, Mixed branes and M(atrix) theory on noncommutative torus, hep-th/9803067; Noncommutative geometry from string and branes, JHEP 9902:016 (1999), preprint hep-th/9810072; Dirac quantization of open string and noncommutativity in branes, Nucl. Phys. B 576 (2000) 578, preprint hep-th/9906161.

N. Seiberg, and E. Witten, String theory and noncommutative geometry, JHEP 9909:032 (1999), preprint hep-th/9908142.

[12] Ali H. Chamseddine, Deforming Einstein's gravity, Phys. Lett. B 504 (2001) 33-37, preprint hep-th/0009153.

Ali. H. Chamseddine, Complex gravity in noncommutative spaces, Commun. Math. Phys. 218 (2001) 283-292, preprint hep-th/0005222.

J. W. Moffat, Noncommutative quantum gravity, Phys. Lett. B 491 (2000) 345-352, preprint hep-th/0007181.

J. W. Moffat, Perturbative noncommutative quantum gravity, Phys. Lett. B 493 (2000) 142-148, preprint hep-th/0008089.

S. I. Vacaru, Gauge and Einstein gravity from non-Abelian gauge models 
on noncommutative spaces, Phys. Lett B 498 (2001) 74-86, preprint hepth/0009163.

V. P. Nair, Gravitional fields on a noncommutative space, hep-th/0112114.

M. Banados, O. Chandia, N. Grandi, F.A. Schaposnik, G.A. Silva Threedimensional noncommutative gravity, Phys. Rev. D64 : 084012, 2001, preprint hep-th/0104264.

[13] R. Jackiw, S. Y. Pi, Covariant coordinate transformations on noncommutative space, Phys. Rev. Lett. 88 (2002) 111603, preprint hep-th/0111122.

S. Cacciatori, A. H. Chamseddine, D. Klemm, L. Martucci, W. A. Sabra, D. Zanon, Noncommutative gravity in two dimensions, hep-th/0203038.

[14] S. Corley, D. Lowe, and S. Ramgoolam, Einstein-Hilbert action on the brane for the bulk graviton, JHEP 0107 (2001) 030, preprint hep-th/0106067. 